\title{
Influence of the Height of Carbon Nanotubes on Hot Switching of Au/Cr-Au/MWCNT Contact Pairs
}

\author{
H. Liu ${ }^{1}$, A.P. Lewis ${ }^{2}$, S.H. Pu ${ }^{1}$, L. Jiang ${ }^{2}$, J.W. McBride ${ }^{1}$ \\ ${ }^{1}$ University of Southampton Malaysia Campus, Nusajaya, 79200, Johor, Malaysia \\ ${ }^{2}$ Faculty of Engineering and the Environment, University of Southampton, Southampton, SO17 1BJ, United Kingdom
}

\{hl1v13, a.p.lewis, SuanHui.Pu, L.Jiang, J.W.McBride\}@ soton.ac.uk

\begin{abstract}
Gold coated Multi-Walled Carbon Nanotube (MWCNT) composites have been used for electrical contact surfaces in previous studies. It was shown that the composite could significantly improve switch lifetime, and exhibited potential as a useful contact material for MEMS switches. The reason is attributed to the contribution of the compressibility of the MWCNT forest, which creates a compliant layer under the gold films. In this study, experiments were performed for samples with different heights of MWCNT forests, namely $30 \mu \mathrm{m}, 50 \mu \mathrm{m}$ and $80 \mu \mathrm{m}$. The influence of MWCNT height on the switching behavior and lifetime were studied. An outcome of the work was that the sample with $80 \mu \mathrm{m}$ height forests showed the longest lifetime, however the composites with $30 \mu \mathrm{m}$ and $50 \mu \mathrm{m}$ forest heights showed lower contact resistances, a parameter of high importance for MEMS switch applications.
\end{abstract}

\section{Introduction}

For MEMS switches, a stable and low contact resistance is required, typically $<1 \Omega[1]$. Researchers have investigated a range of materials to improve the reliability of MEMS switches whilst retaining low electrical contact resistance. Commonly used materials for ohmic contacts include gold, palladium and platinum [1], ruthenium [2] and rhodium [3], and gold-alloys [4], but these materials are relatively soft and therefore wear easily. Other wear-resistant materials like silicon carbide and diamond films have low electrical conductivity and are therefore unsuitable for electrical contact application [5]. The application of multi-walled carbon nanotubes (MWCNT) as contact surfaces was first investigated in [6], and was later improved to incorporate a gold-coated surface; i.e. a forest of MWCNT forms a compliant layer underneath the metallic film, normally gold.

Individual MWCNT shows a very high elastic modulus [79], but when grown as a vertically aligned forest, they produce a compliant mechanical surface, with the effective modulus reduced by several orders of magnitude [10-11]. Vertical aligned MWCNT forest shows super-compressible behavior [11], this high compliance of MWCNT can be utilized to spread the applied mechanical load which was created during the closing process, to reduce the damage to the contact surfaces.

Nano-indentation technique has been used to measure the mechanical properties and the effective modulus of vertically aligned MWCNT films, and it was shown that the effective elastic modulus decreased as the height of MWCNT increased [12-13]. Furthermore, initial nano-indentation studies have been conducted to study the mechanical behavior of gold coated MWCNT composite (Au/MWCNT) [14-15].
During the initial testing using Au/MWCNT composite as electrical contact surfaces, samples with a MWCNT height of $50 \mu \mathrm{m}$ were used by Yunus et al. [6]; this work showed that the use of Au/MWCNT as a contact surface could provide a stable contact resistance. This work was further developed by Chianrabutra et al [16-17] to consider the effect of MWCNT forests with heights of $30 \mu \mathrm{m}$. It was shown that the contact resistance remained stable for a large number of switching cycles (between 80 to 120 million switching cycles with current levels of 20 to $50 \mathrm{~mA}$ ). This showed that a $\mathrm{Au} / \mathrm{MWCNT}$ surface had potential as a contact material for MEMS switches. There were differences in the experimental setup reported by Yunus et al. and Chianrabutra et al. which meant that no comparison could be made with different heights of MWCNT forests. An aim of this work is to investigate and discuss the influence of height of MWCNT on the contact behavior and switching lifetime.

Three samples with different heights of MWCNT namely $30 \mu \mathrm{m}, 50 \mu \mathrm{m}$ and $80 \mu \mathrm{m}$ are studied in the paper, and the comparisons are made in terms of the contact resistance, the number of contact bounces and the switching lifetime.

\section{Preparation of contact pairs}

The fabrication of the contact pairs has been discussed in previous study $[16-17,14]$. The cathode was a $2 \mathrm{~mm}$ diameter stainless steel hemisphere sputter coated with a $10 \mathrm{~nm}$ and 500 $\mathrm{nm}$ thick $\mathrm{Cr}$ and $\mathrm{Au}$ layer, respectively; this results in a $\mathrm{Cr} / \mathrm{Au}$ contact surface. The anode was a gold coated MWCNT forest which is grown on a silicon substrate. To fabricate the $\mathrm{Au} / \mathrm{MWCNT}$ composites, a buffer layer of $\mathrm{Al}_{2} \mathrm{O}_{3}$ and a catalyst layer of $\mathrm{Fe}$ were sputtered on silicon wafer which had a $1 \mu \mathrm{m}$ oxide layer. Following this the vertically aligned MWCNT were grown using thermal chemical vapor deposition (CVD). In the CVD process, the flow rate of gaseous carbon source ethylene $\left(\mathrm{C}_{2} \mathrm{H}_{4}\right)$ was kept constant at $0.3 \mathrm{slm}$, and the growth temperature was fixed at $875^{\circ} \mathrm{C}$. The growth time was used to control the height of MWCNT. Three heights of MWCNT were grown, namely $30 \mu \mathrm{m}, 50 \mu \mathrm{m}$ and $80 \mu \mathrm{m}$. After the growth of the MWCNT forests, the final step was to sputter them with $500 \mathrm{~nm}$ of gold.

\section{Experimental conditions}

The experimental apparatus is the same as described in [16-17], and a schematic of the testing system is shown in Figure 1. To simulate the repeated switching behavior of MEMS switches, the Au/MWCNT composite was attached to a PZT cantilever which was actuated by a function generator. A 4-probe micro-ohmmeter was used to measure the contact resistance. All experiments were conducted with load voltage of $4 \mathrm{~V}$.

The study firstly investigated the switching behavior over a range of contact forces from $0.2 \mathrm{mN}$ to $1 \mathrm{mN}$. The 
experiments were performed at low current (1 mA) to reduce thermal effects, due to Joule heating, on contact behavior. The testing frequency was $2 \mathrm{~Hz}$. The number of bounces, $B_{\text {test }}$ was recorded during tests, while the contact resistance $R_{\mathrm{c}}$ was read using the 4-probes measurement method, and the values were recorded after the PZT cantilever was stopped for 3 minutes, ensuring the contact was stable. The experiments were repeated at three different locations for each sample.

The lifetime testing was conducted with the contact force fixed at $1 \mathrm{mN}$; the load currents tested were $100 \mathrm{~mA}$ and $200 \mathrm{~mA}$. The contact resistance was measured after the $10^{\text {th }}$, $100^{\text {th }}, 1000^{\text {th }}, 10000^{\text {th }}$ and $100000^{\text {th }}$ switching cycles, and then periodically until the contact failed. The testing frequency was $2 \mathrm{~Hz}$ for $10^{\text {th }}$ and $100^{\text {th }}$ cycles, and $30 \mathrm{~Hz}$ from the $1000^{\text {th }}$ cycles onwards. As described above for the $1 \mathrm{~mA}$ case, the values of $R_{\mathrm{c}}$ were recorded 3 minutes after pausing the experiment. As bouncing results in additional opening and closing events during cycling, the number of bounces should be considered while counting the total number of switching events, the number of total switching events can be calculated by:

$$
N_{\text {total }}=\sum_{k} t_{k} f_{k} B_{k}
$$

Where $k$ denotes the testing period between two pausing times. $t, f, B$ represents the testing time in seconds, the testing frequency and the number of bounces during each testing period, respectively.

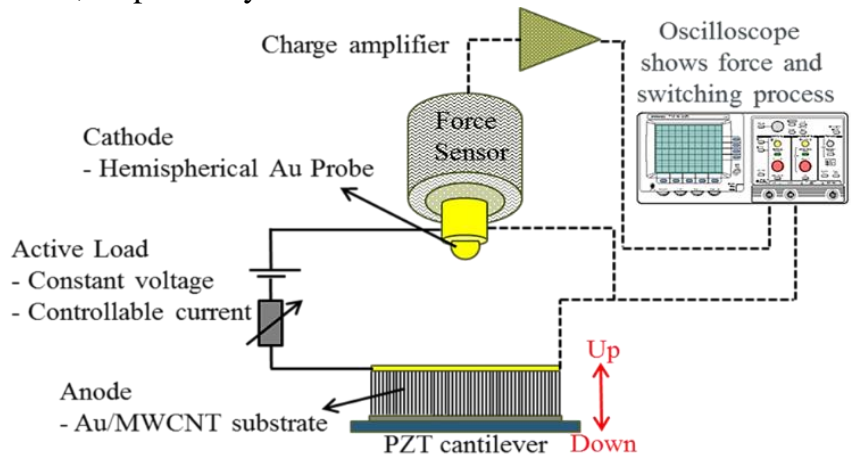

Figure 1. Schematic of experimental setup. [17]

\section{Results and discussion}

\section{A. Initial cycling with low current}

The averaged results of $R_{\mathrm{c}}$ and $B_{\text {test }}$ as a function of applied force and with the low current level of $1 \mathrm{~mA}$ are plotted in Figure 2 and Figure 3 respectively. Due to the much higher contact resistance $(>1.5 \Omega$ ) with $80 \mu \mathrm{m}$ MWCNT, two horizontal axis were used to show clearly the resistance values, as shown in Figure 2, where the left horizontal axis is for sample with $80 \mu \mathrm{m} \mathrm{MWCNT}$, and the right axis is for samples with $30 \mu \mathrm{m}$ and $50 \mu \mathrm{m}$ MWCNT. As expected, the contact resistance decreased with an increase in the applied contact force. An unexpected outcome was that the contact resistance of the sample with $80 \mu \mathrm{m}$ MWCNT forests was significantly higher than for the 30 and $50 \mu \mathrm{m}$ samples. The nano-indentation tests with Au/MWCNT composite showed that the elastic modulus and hardness of the composite decreased with the height of MWCNT [14], therefore larger deformation and greater contact area and thus smaller contact resistance were expected for the composite with longer
MWCNT, as observed for the samples with $30 \mu \mathrm{m}$ and $50 \mu \mathrm{m}$ MWCNT. An explanation for the unexpected result could be attributed to the surface roughness (shown in Table 1). The roughness data were measured using a laser profiler (Taicaan XYRIS 4000CL). The scan was performed over an area of $1 \times 1 \mathrm{~mm}^{2}$ with $51 \times 51$ scanning points of data, thus the interval space between points was $2 \mu \mathrm{m}$. The values of roughness parameters $R a$ and $R q$ were recorded from three profile lines 'Horizontal', 'Vertical' and 'Free' (diagonal) and then averaged. The sample with $80 \mu \mathrm{m}$ MWCNT showed a much rougher surface than the 30 and $50 \mu \mathrm{m}$ MWCNT samples. A rougher surface would be expected to result in a smaller effective contact area and therefore an increase in the contact resistance [18].

It was observed that the number of bounces decreased with the contact force. An explanation for this is that the elastic deformation dominates at low forces and therefore the composite exhibits spring behavior, then with contact force increases, plastic deformation appears and becomes more dominant, thus the number of bounces decreases. However, the influence of height of MWCNT on the number of bounces does not show a regular trend (Figure 3 ). Indeed, as shown in Figure 4, the nanoindentation tests showed that the height of MWCNT dominates the effect of the residual depth of the impact, but has little influence on the energy absorption [14], thus little impact on the number of bounces.

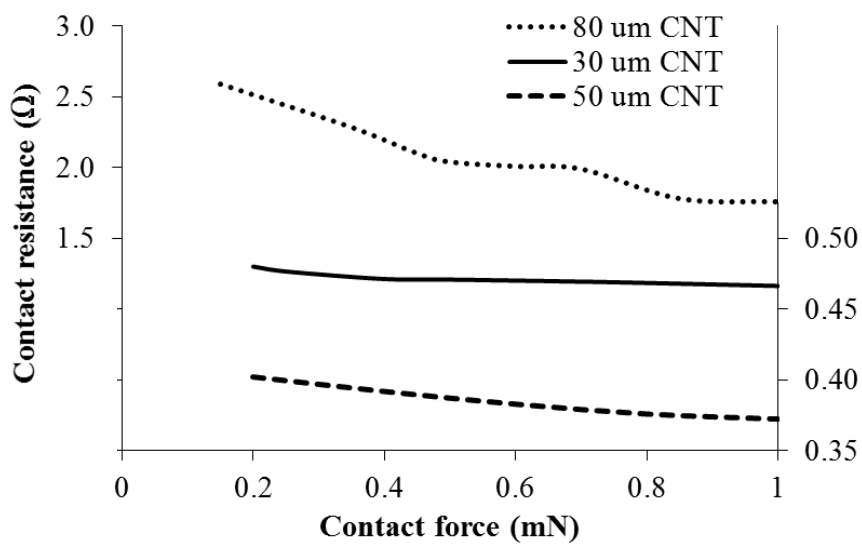

Figure 2. Contact resistance as a function of force for samples with different heights MWCNT, with current of $1 \mathrm{~mA}$.

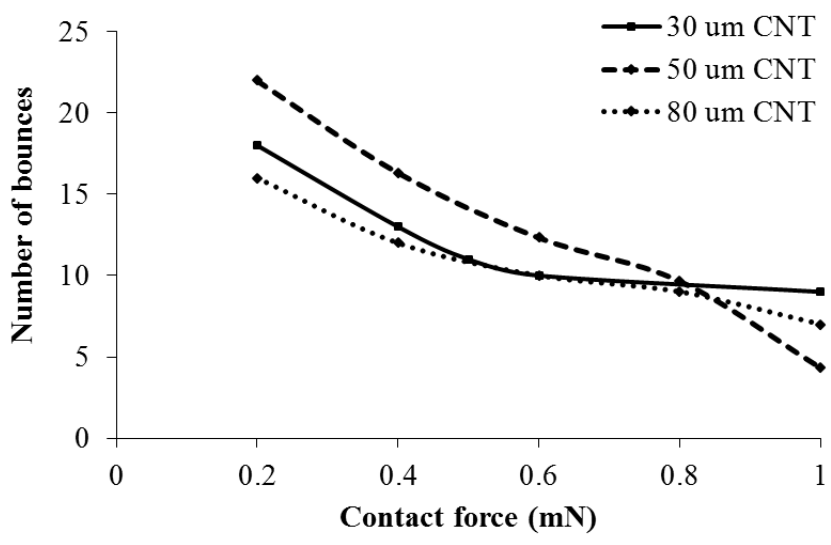

Figure 3. Number of bounces as a function of force for samples with different heights MWCNT, the current was $1 \mathrm{~mA}$ and testing frequency was $2 \mathrm{~Hz}$. 


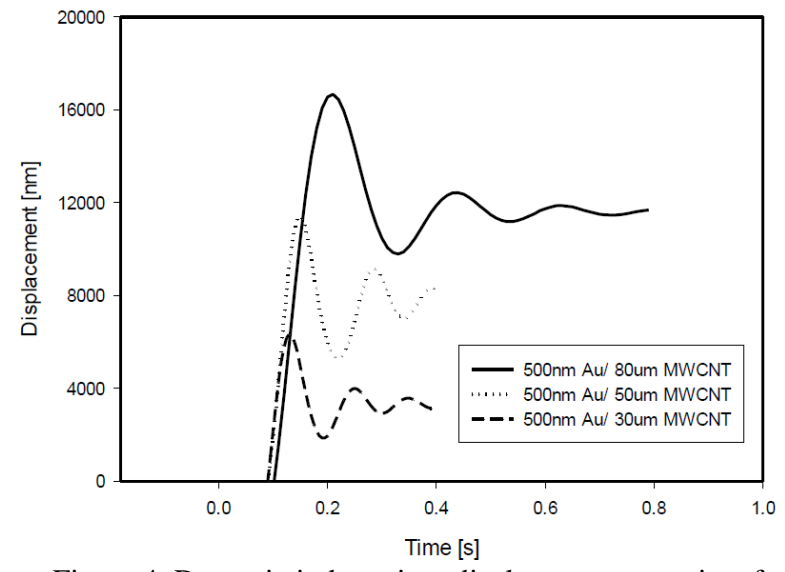

Figure 4. Dynamic indentation: displacement over time for $1 \mathrm{mN}$ indent, for composite with varied MWCNT heights (after [14]).

Table 1. Roughness data for surfaces of gold coated on different heights MWCNT.

\begin{tabular}{|c|c|c|c|}
\hline Sample & $\begin{array}{c}\text { MWCNT Height } \\
(\mu \mathrm{m})\end{array}$ & $\begin{array}{c}\boldsymbol{R a} \\
(\mu \mathrm{m})\end{array}$ & $\begin{array}{c}\boldsymbol{R} \boldsymbol{q} \\
(\mu \mathrm{m})\end{array}$ \\
\hline 1 & 30 & 1.3933 & 1.48767 \\
\hline 2 & 50 & 1.07133 & 1.46167 \\
\hline 3 & 80 & 2.39767 & 2.97167 \\
\hline
\end{tabular}

\section{B. Lifetime test results}

Figure 5 and Figure 6 show the contact resistance versus the number of cycles for $100 \mathrm{~mA}$ and $200 \mathrm{~mA}$ respectively. It was assumed in this study that a contact has failed when the contact resistance increased beyond three times the nominal value. However, as also shown in a previous study [16], the contact resistance tended to increase abruptly during the failure stage, hence some of the final values of contact resistance recorded were significantly larger than 3 times nominal contact resistance.

With a load current of $100 \mathrm{~mA}$, as shown in Figure 5, it was found that the sample with $80 \mu \mathrm{m}$ in height MWCNT survived the greatest number of cycles; failure occurred after 65.55 million switching events. Following this, the sample with $30 \mu \mathrm{m}$ MWCNT then the sample with $50 \mu \mathrm{m}$ MWCNT. The sample with a $50 \mu \mathrm{m}$ forest had a lifetime of 32.23 million switching cycles. With a load current of $200 \mathrm{~mA}$, as shown in Figure 6, once again the sample with $80 \mu \mathrm{m}$ in height MWCNT forest survived for the greatest number of cycles (12.86 million cycles). However, the sample with 30 $\mu \mathrm{m}$ showed the shortest lifetime and failed after 1.3 million cycles, this value is the same order as reported in [17], where the contact failed after 4.8 million cycles.

The nano-indentation test [14] revealed that the composite with $80 \mu \mathrm{m}$ MWCNT had a much higher contact compliance than the two others (as shown in Figure 7). The contact compliance is defined as the inverse of stiffness, and is inverse proportional to the elastic modulus for a given composite and boundary conditions. The higher compliance can help to reduce the damage caused by the mechanical impact of the contacting surfaces. The compliance of the composite with 50 $\mu \mathrm{m}$ MWCNT is a little larger than the $30 \mu \mathrm{m}$ MWCNT, but the surface of composite with $50 \mu \mathrm{m}$ MWCNT is not very uniform (see Figure 9) and it might cause shortest lifetime at current level of $100 \mathrm{~mA}$.

Comparing Figure 5 and Figure 6, it was found that the contacts failed sooner with a load current of $200 \mathrm{~mA}$ than with $100 \mathrm{~mA}$. This agrees with the results observed in [17]: an increase in the load current results in an increase in the rate of fine transfer and hence the failure of the contact area will be reached sooner.

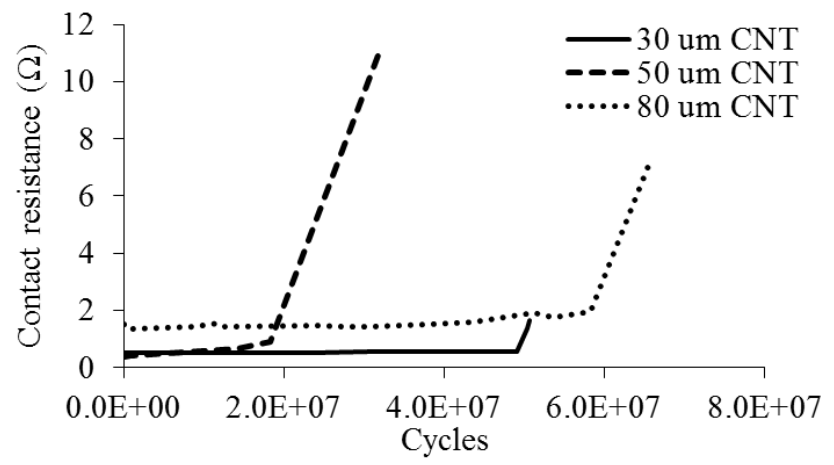

Figure 5. Contact resistance over the total number of cycles with current of $100 \mathrm{~mA}$ for samples of $500 \mathrm{~nm}$ gold coated on different heights MWCNT.

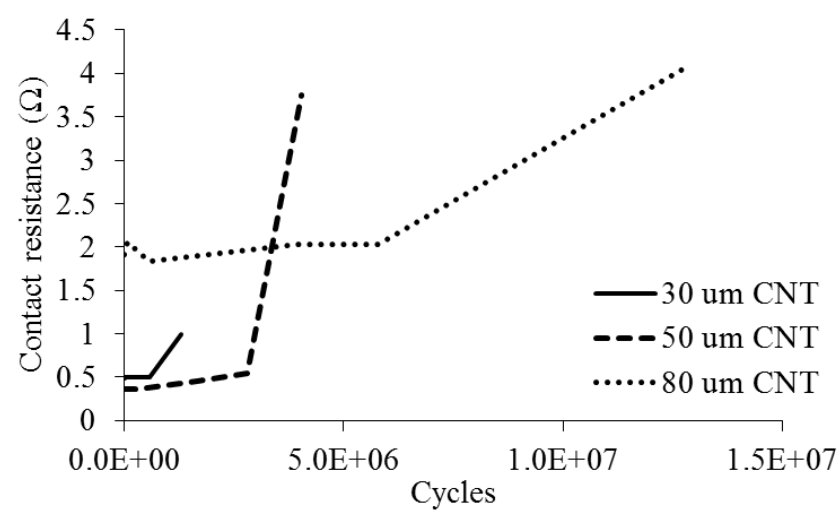

Figure 6. Contact resistance over the total number of cycles with current of $200 \mathrm{~mA}$ for samples of $500 \mathrm{~nm}$ gold coated on different heights MWCNT.

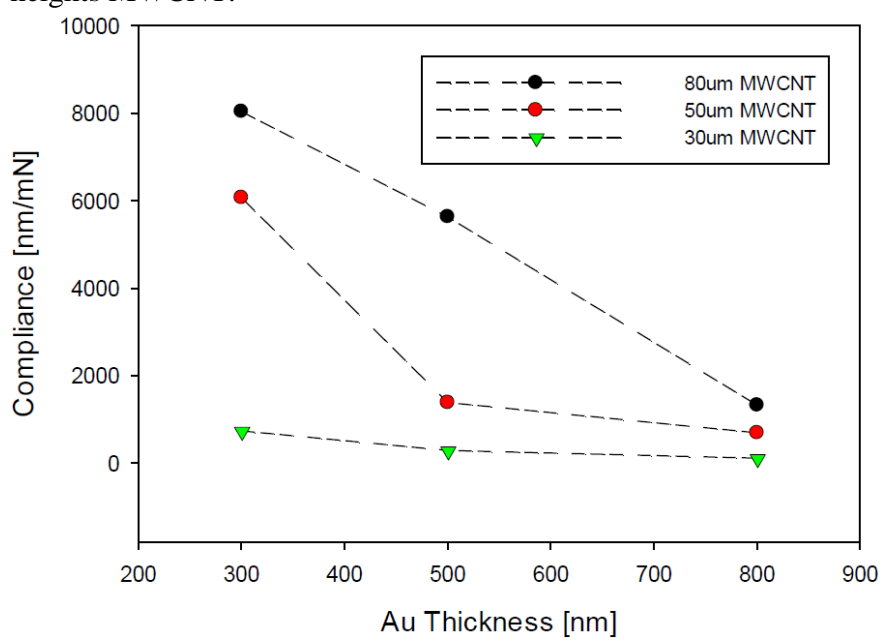

Figure 7. The measured compliance for the samples with different layer configurations (after [14]).

The SEM images of the failed contacts with load currents of $100 \mathrm{~mA}$ are shown in Figure 8- Figure 10 for samples with 
$30 \mu \mathrm{m}, 50 \mu \mathrm{m}$ and $80 \mu \mathrm{m}$ MWCNT respectively. The images indicates a similar wear process as discussed in [16] and [17]: the failure is a combination of the effect of fine transfer process and delamination. A fitting circle was used to brace the failed area. It was shown that the diameter of fitting circle was larger with taller MWCNT forests. This matched the results from the nano-indentation tests that the hardness of Au/MWCNT decreased with MWCNT height [14], thus the contact area was larger. In addition, for the sample with 80 $\mu \mathrm{m}$ MWCNT, due to its much rougher surfaces, contact was made only at some of the higher asperities, the contact area was not completely flattened even after millions of switching cycles.

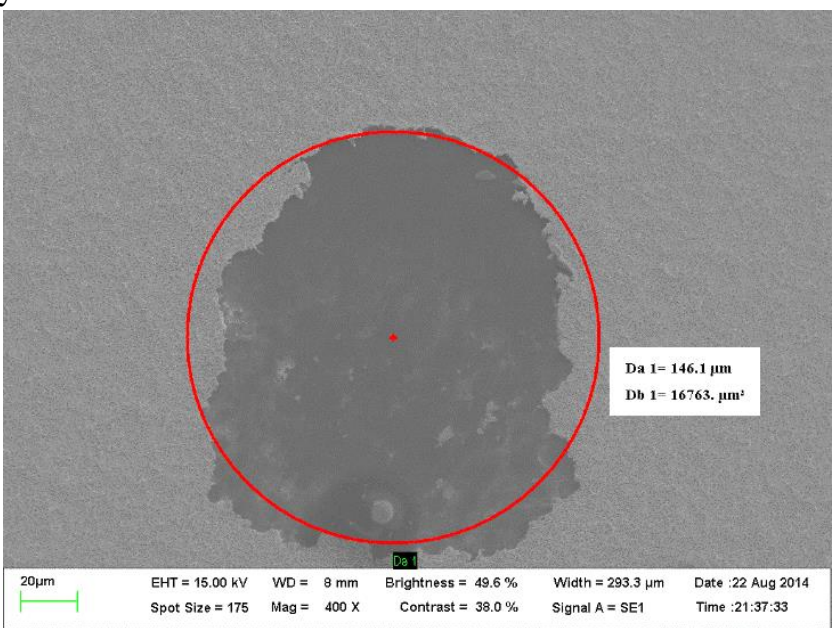

Figure 8. Au-Au/MWCNT surface of the composite $500 \mathrm{~nm}$ $\mathrm{Au} / 30 \mu \mathrm{m}$ MWCNT after testing with a current of $100 \mathrm{~mA}$. The diameter of the fitting circle is around $146 \mu \mathrm{m}$.

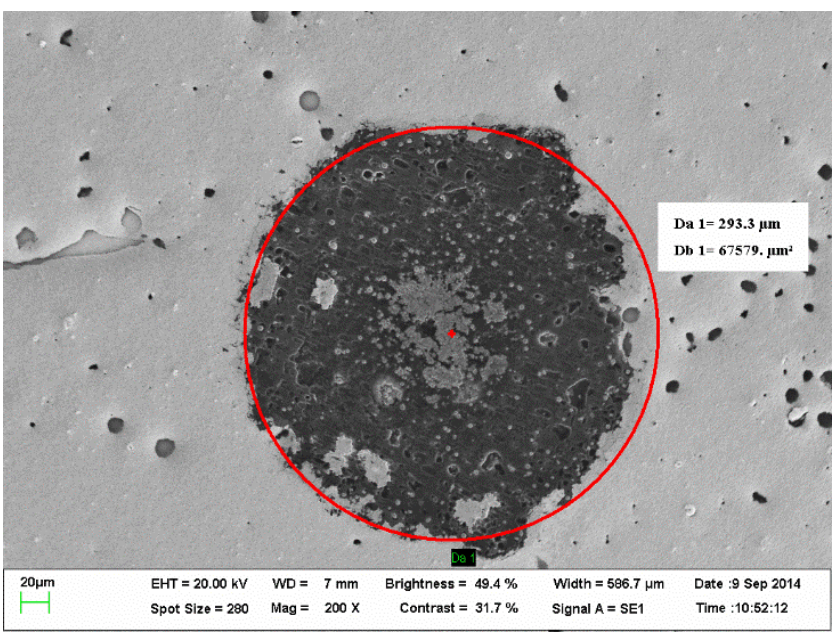

Figure 9. Au-Au/MWCNT surface of the composite $500 \mathrm{~nm}$ $\mathrm{Au} / 50 \mu \mathrm{m}$ MWCNT after testing with a current of $100 \mathrm{~mA}$. The diameter of the fitting circle is around $293 \mu \mathrm{m}$.

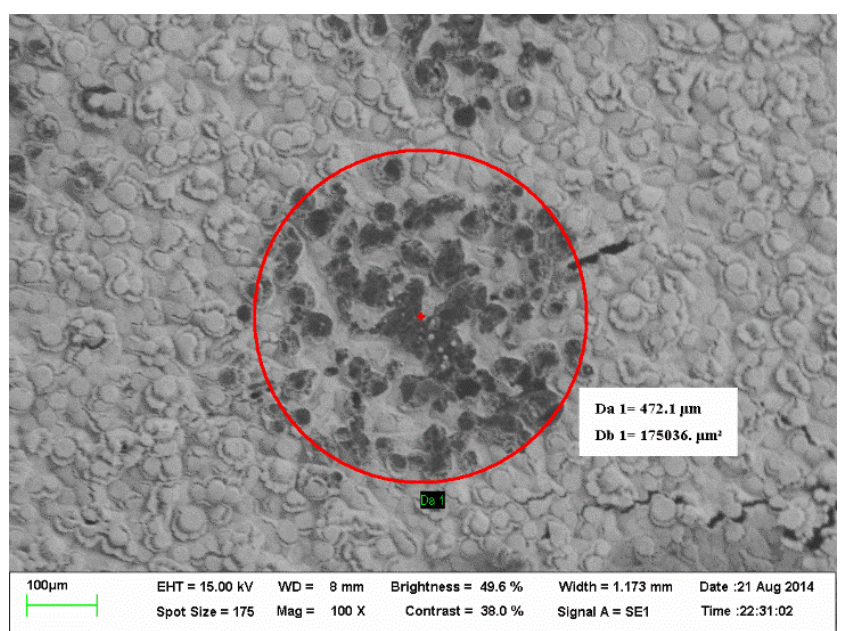

Figure 10. Au-Au/MWCNT surface of the composite $500 \mathrm{~nm}$ $\mathrm{Au} / 80 \mu \mathrm{m}$ MWCNT after testing with a current of $100 \mathrm{~mA}$. The diameter of the fitting circle is around $472 \mu \mathrm{m}$.

\section{Conclusions}

Three heights of MWCNT forests were used for gold coated MWCNT composites as electrical contact surfaces. The samples with $30 \mu \mathrm{m}$ and $50 \mu \mathrm{m}$ MWCNT showed similar contact resistance and lifetime behavior, whilst the sample with $80 \mu \mathrm{m}$ MWCNT exhibited a much higher contact resistance but longer lifetime. The shorter MWCNT forests showed smoother surfaces and lower values of contact resistance. Therefore these results indicate that by controlling the height of the MWCNT forest it is possible to introduce a trade-off between lifetime and contact resistance for Au/MWCNT composites.

\section{Acknowledgments}

This work was supported by the Innovative Electronics Manufacturing Research Centre (IeMRC) and Engineering and Physical Sciences Research Council (EPSRC) under grant number: EP/H03014X/1, and Ministry of Education Malaysia grant under number: FRGS-12014-TK01-USMC-1. The authors would also thank C. Chianrabutra and M.P. Down for the kind help on the test rigs and providing samples for the experiments.

\section{References}

1. R. A. Coutu, J. R. Reid, R. Cortez, R. E. Strawser, and P. E. Kladitis, "Microswitches with sputtered Au, AuPd,Auon-AuPt, and AuPtCu alloy electric contacts," IEEE Trans. Compon. Packag. Technol., Vol. 29, No. 2, pp. 341-349, 2006.

2. F. Ke, J. Miao, and J. Oberhammer, "A Ruthenium-Based Multimetal-Contact RF MEMS Switch With a Corrugated Diaphragm," J. Microelectromechanical Syst., Vol. 17, No. 6, pp. 1447 -1459, 2008.

3. A. Broue, et al, "Multi-Physical Characterization of Micro-Contact Materials for MEMS Switches," Proc 56th IEEE Holm Conference on Electrical Contacts, Charleston, SC, 2010, pp. 1-10.

4. B. F. Toler, R. A. Coutu, and J. W. McBride, "A review of micro-contact physics for microelectromechanical systems (MEMS) metal contact switches," J. Micromechanics Microengineering, Vol. 23, No. 10, p. 103001, 2013. 
5. J. W. McBride, E. M. Yunus, and S. M. Spearing, "Improving the contact resistance at low force using gold coated carbon nanotube surfaces," Eur. Phys. J. - Appl. Phys., Vol. 50, No. 01, p. 12904 (6 pages), 2010.

6. E. M. Yunus, J. W. McBride, and S. M. Spearing, "The Relationship Between Contact Resistance and Contact Force on Au-Coated Carbon Nanotube Surfaces Under Low Force Conditions," IEEE Trans. Compon. Packag. Technol., Vol. 32, No. 3, pp. 650-657, 2009.

7. H. J. Qi, K. B. K. Teo, K. K. S. Lau, M. C. Boyce, W. I. Milne, J. Robertson, and K. K. Gleason, "Determination of mechanical properties of carbon nanotubes and vertically aligned carbon nanotube forests using nanoindentation," $J$. Mech. Phys. Solids, Vol. 51, No. 11-12, pp. 2213-2237, 2003.

8. B. I. Yakobson and P. Avouris, "Mechanical Properties of Carbon Nanotubes," Carbon Nanotubes, Springer Berlin Heidelberg, 2001, pp. 287-327.

9. R. S. Ruoff, D. Qian, and W. K. Liu, "Mechanical properties of carbon nanotubes: theoretical predictions and experimental measurements," Comptes Rendus Phys., Vol. 4, No. 9, pp. 993-1008, 2003.

10. N. J. Ginga, W. Chen, and S. K. Sitaraman, "Waviness reduces effective modulus of carbon nanotube forests by several orders of magnitude," Carbon, Vol. 66, pp. 57-66, 2014.

11. A. Cao, P. L. Dickrell, W. G. Sawyer, M. N. GhasemiNejhad, and P. M. Ajayan, "Super-Compressible Foamlike Carbon Nanotube Films," Science, Vol. 310, No. 5752, pp. 1307-1310, 2005.

12. Y. Gao, T. Kodama, Y. Won, S. Dogbe, L. Pan, and K. E. Goodson, "Impact of nanotube density and alignment on the elastic modulus near the top and base surfaces of aligned multi-walled carbon nanotube films," Carbon, Vol. 50, No. 10, pp. 3789-3798, 2012.

13. K. Eom, K. Nam, H. Jung, P. Kim, M. S. Strano, J.-H. Han, and T. Kwon, "Controllable viscoelastic behavior of vertically aligned carbon nanotube arrays," Carbon, Vol. 65, pp. 305-314, 2013.

14. M. P. Down, R. Cook, L. Jiang, and J. W. McBride, "Mechanical Characterisation and Optimisation of Carbon Nanotube Composite Surfaces for Electrical Contact," Proc 27th International Conference on Electrical Contacts (ICEC 2014), Dresden, Germany, Jun. 2014, pp. 1-6.

15. H. Liu, M. P. Down, J. W. McBride, S. H. Pu, and L. Jiang, "Mechanical Characterization of a $\mathrm{Au}$ Coated Carbon Nanotube Multi-layered Structure," Proc 60th IEEE Holm Conference on Electrical Contacts (Holm), New Orleans, LA, USA, Oct. 2014, pp 1-8.

16. J. W. McBride, C. Chianrabutra, L. Jiang, and S. H. Pu, "The contact resistance performance of gold coated carbon-nanotube surfaces under low current switching," Proc 12th International Session on Electro-Mechanical Devices (IS-EMD2012), Chiba, JP, 2012, p. 8pp.

17. C. Chianrabutra, L. Jiang, A. P. Lewis, and J. W. McBride, "Evaluating the Influence of Current on the Wear Processes of $\mathrm{Au} / \mathrm{Cr}-\mathrm{Au} / \mathrm{MWCNT}$ Switching Surfaces," Proc 59th IEEE Holm Conference on Electrical Contacts (HOLM), Newport, RI, USA, Sep. 2013, pp. 1-6.
18. P. Sahoo and N. Ghosh, "Finite element contact analysis of fractal surfaces," J. Phys. Appl. Phys., Vol. 40, pp. 4245-4252, 2007. 